\title{
Who seeks genetic susceptibility testing for Alzheimer's disease? Findings from a multisite, randomized clinical trial
}

J. Scott Roberts, PhD ${ }^{1}$, Melissa Barber, ScM ${ }^{3}$, Tamsen M. Brown, MS ${ }^{1,2}$, L. Adrienne Cupples, PhD ${ }^{4}$, Lindsay A. Farrer, PhD ${ }^{1,2,4,5}$, Susan A. LaRusse, $M S^{6}$, Stephen G. Post, $P h D^{7}$, Kimberly A. Quaid, PhD ${ }^{8}$, Lisa D. Ravdin, PhD ${ }^{9}$, Norman R. Relkin, $M D, P h D^{9}$, A. Dessa Sadovnick, PhD ${ }^{10}$, Peter J. Whitehouse, $M D, P h D^{3}$, John L. Woodard, $P h D^{11}$ and Robert C. Green, $M D, M P H^{1,2,5}$

\begin{abstract}
Purpose: Alzheimer's disease, for which one form of the apolipoprotein E (APOE) genotype is a risk factor, provides a paradigm in which to examine response to susceptibility testing for common, complex diseases. This study's main purposes were to estimate interest in such testing and to examine demographic predictors of study participation. Methods: In this 3-site, randomized clinical trial (RCT), the intervention was a risk assessment program wherein genetic counselors educated adult children of AD patients about lifetime risk of disease based on their gender, family history, and APOE genotype. Two groups of participants were followed from initial contact to RCT enrollment: those who were systematically contacted through research registries, and those who were self-referred. Results: Of 196 systematically contacted participants, 47, or 24\%, progressed from initial contact to RCT enrollment. These participants were more likely to be below age 60 (adjusted OR $=3.83, P<0.01$ ) and college educated (adjusted $\mathrm{OR}=3.48, P<0.01$ ). Of 179 self-referred participants, 115 , or $64 \%$, progressed from initial contact to RCT enrollment. Most self-referred participants had a college education and were female (79\%). Conclusions: In the first RCT to examine genetic susceptibility testing for AD, uptake rates were sufficiently high to merit concern that future test demand may strain available education and counseling resources. Findings suggest that susceptibility testing for $A D$ may be of particular interest to women, college educated persons, and persons below age 60. Genet Med 2004:6(4):197-203.
\end{abstract}

Key Words: genetic testing, Alzheimer's disease, test uptake, APOE, genetic counseling

Advances in genetic research have led to an increased number of testing procedures to determine future risk of disease among at-risk individuals. In Huntington's disease (HD), for example, presence of a genetic mutation means almost certain development of the disease later in life. However, an increasing number of genes are being identified that confer susceptibility for a given disease rather than inevitably causing it. Given that

\footnotetext{
From the Departments of ${ }^{I}$ Neurology and ${ }^{2}$ Medicine (Genetics Program), Boston University School of Medicine, Boston, Massachusetts; ${ }^{3}$ University Memory and Aging Center, Case Western Reserve University/University Hospitals of Cleveland, Ohio; Departments of ${ }^{4}$ Biostatistics and ${ }^{5}$ Epidemiology, Boston University School of Public Health, Boston, Massachusetts; ${ }^{6}$ Taub Institute for Research on Alzheimer's Disease and the Aging Brain, Columbia University, New York, NY; ${ }^{7}$ Department of Biomedical Ethics, Case Western Reserve University School of Medicine; ${ }^{8}$ Department of Medical and Molecular Genetics, Indiana University School of Medicine; ${ }^{9}$ Department of Neurology and Neuroscience, Weill Medical College of Cornell University; ${ }^{10}$ Department of Neuropsychiatric Genetics, University of British Columbia; ${ }^{11}$ Department of Psychology, Rosalind Franklin University of Medicine and Science

Scott Roberts, PhD, Boston University Alzheimer's Disease Center, 715 Albany Street B-7800, Boston, MA 02118 .

Received: March 4, 2004.

Accepted: March 29, 2004.
}

DOI: 10.1097/01.GIM.0000132688.55591.77 such genes may provide risk information for common diseases (e.g., stroke, depression),${ }^{1-2}$ there is a growing need to understand how at-risk populations might respond to the option of genetic susceptibility testing. A prominent case in point is Alzheimer's disease (AD).

Recent advances in genetic research on AD have brought about the possibility of genetic susceptibility testing for asymptomatic individuals. ${ }^{3-4}$ The apolipoprotein E (APOE) $\epsilon 4$ allele on chromosome 19 is the only susceptibility gene for $\mathrm{AD}$ that has been widely confirmed to date, although several others are under investigation. ${ }^{5-6}$ Although the presence of $\epsilon 4$ allele(s) increases risk of $\mathrm{AD}$ up to 15 -fold compared to other APOE genotypes, it is neither necessary nor sufficient to cause the disease. ${ }^{7}$ As such, APOE is distinct from the very rare mutations (i.e., amyloid precursor protein [APP], presenilin 1 and 2 genes) that almost inevitably cause $\mathrm{AD}$, typically with earlier than usual onset. ${ }^{8}$ Susceptibility testing for AD therefore differs in important ways from predictive testing for disease-causing genes; it is relevant to a much larger at-risk population yet provides much less certain risk information than predictive testing. ${ }^{9}$ This limitation, coupled with a general lack of treatment options for $\mathrm{AD}$, has prompted several consensus state- 
ments cautioning against the premature introduction of clinical susceptibility testing in asymptomatic individuals. ${ }^{10-13}$ However, given that treatment advances, patient demand, and clinical trials seeking "enriched" at-risk samples could make susceptibility testing an increasingly relevant risk assessment option, there is a growing need to examine genetic susceptibility testing in a research context. ${ }^{14}$ To anticipate response to genetic testing for $\mathrm{AD}$, it is important to evaluate the level of interest in such testing, as well as to determine factors that are associated with test-seeking.

To date, interest in genetic testing for $\mathrm{AD}$ has only been examined through surveys posing hypothetical scenarios. A survey of 203 first-degree relatives of persons with AD in Michigan found that a majority of participants expressed interest in seeking genetic testing, with the following demographic, attitudinal, and psychological variables predicting such interest: (1) male gender; (2) more active health information-seeking style; (3) greater perceived threat of $\mathrm{AD}$; and (4) rating of test benefits as more important than its limitations and risks. ${ }^{15} \mathrm{~A}$ similar survey of a racially diverse convenience sample in the Southeast found generally high interest $(69 \%)$ in a hypothetical genetic test for AD. ${ }^{16}$ A comparison of White and African American respondents in this survey found that Whites expressed greater interest in testing and endorsed more reasons for seeking it. ${ }^{17}$ Using a general population telephone survey, Neumann et al. ${ }^{18}$ found that reported inclination to take a hypothetical predictive test for $\mathrm{AD}$ was generally constant across gender, education, and race categories. Although these findings suggest widespread interest in genetic testing for $\mathrm{AD}$, it is well known that pretest intentions are not good predictors of actual utilization of testing. For example, the vast majority of people at risk for the autosomal dominant Huntington's disease expressed interest in testing in pretest surveys, but only a fraction of such individuals presented for testing. ${ }^{19}$ It is therefore more relevant to examine predictors of test-seeking in a "real-life" test situation.

\section{MATERIALS AND METHODS}

\section{Overview}

The REVEAL Study (Risk Evaluation and Education for Alzheimer's Disease) is the first randomized controlled trial designed to evaluate the impact of risk assessment, using APOE genotype disclosure, for $\mathrm{AD}$. The study protocol was developed by a multidisciplinary team of experts in the fields of Alzheimer's disease, neurology, genetics, genetic counseling, psychology, and bioethics. Development of the protocol was overseen and approved by a study External Advisory Board, as well as institutional review boards at each of the three study sites. All participants gave written informed consent at each of the study's three prerandomization phases. We report in this study on factors associated with enrollment in the clinical trial. Findings regarding the impact of susceptibility testing for $\mathrm{AD}$ (e.g., its effects on psychological well-being, health behaviors, insurance choices) will be the subject of future papers and are generally beyond the scope of this article.

\section{Participants}

All participants were adult children of a person with clinically diagnosed and/or autopsy-confirmed AD. Participants were enrolled in the study in one of two ways. The "self-referred" group volunteered for the study after hearing about it in memory assessment clinics, the media, or in public presentations. The "systematically contacted" group was contacted through their family's membership in AD research registries at each of the three study sites (Boston, New York, and Cleveland). These registries consist of patients and family members who have agreed to be contacted about research studies on AD. The Boston and Cleveland registries serve as the main research registries for federally funded Alzheimer's Disease Centers at these sites.

\section{Procedures}

Systematically contacted participants were contacted through their family's membership in research registries. In cases where an adult child was not directly listed in the registry, initial contact was through a mailing to the affected parent's primary caregiver (e.g., spouse). Once a potentially eligible participant was identified, an initial telephone contact took place, during which the rationale and procedures of the study were briefly explained and questionnaires were administered to assess demographic information and attitudes toward $\mathrm{AD}$ and genetic testing. Participants interested in learning more were invited to attend a formal Education Session conducted by the site's genetic counselor.

At the Education Session, the genetic counselor provided information about $\mathrm{AD}$ and the study protocol using a standard slide show presentation. The genetic counselor stressed the distinction between susceptibility and more predictive types of testing for $\mathrm{AD}$, comparing $\mathrm{APOE}$ to rare disease-causing mutations such as APP and the presenilin genes. A featured slide in the slide show read, "APOE testing can be one component of Alzheimer risk assessment, but APOE alone is not a clear predictor of AD," and participants were informed that "not everyone with an $\epsilon 4$ gene will develop Alzheimer's disease, [and] not everyone who develops Alzheimer's disease has an $\epsilon 4$ gene." Also discussed were the possible benefits and limitations of susceptibility testing. Benefits included information to guide future planning and possible reduction in anxiety about developing $\mathrm{AD}$, whereas limitations included the imperfect nature of test information, the lack of treatment options to prevent or cure $\mathrm{AD}$, and a possible increase in anxiety about developing AD. After the Education Session, interested participants progressed to the Counseling/Blood Draw stage of the study, which involved individualized genetic counseling and blood draw for the purposes of APOE genotyping. At this stage, potential participants were also screened to evaluate their neuropsychological functioning and psychiatric status, using the Repeatable Battery for the Assessment of Neuropsychological Status [RBANS],,$^{20}$ Center for Epidemiological Studies-Depression scale $[\mathrm{CES}-\mathrm{D}],{ }^{21}$ and Beck Anxiety Inventory $[\mathrm{BAI}] .{ }^{22}$ All clinical trial participants met inclusionary criteria for $\operatorname{cog}^{-}$ 
nitive (within 1.5 standard deviation of age-matched means on the RBANS) and psychiatric functioning (CES-D cutoff score $=27$; BAI cutoff score $=21$ ). No systematically contacted participants were excluded due to cognitive or psychiatric difficulties. Nine self-referred participants were excluded due to cognitive difficulties, and one was excluded due to psychiatric difficulties. Each of these participants was referred for further clinical assessment. In addition, one self-referred participant died during the course of the study. These 10 self-referred participants were excluded from study analyses because they did not have the opportunity to participate in the clinical trial.

Participants were randomized to either the Intervention or Control Arm of the study. Participants randomized to the Intervention Arm received genetic counseling and risk assessment based on their gender, family history of $\mathrm{AD}$, and $\mathrm{APOE}$ genotype, whereas those randomized to the Control Arm received genetic counseling and risk assessment based only on their gender and family history. In each Arm, the genetic counselor met individually with participants in 30- to 60-minute risk disclosure sessions to communicate risk, provide support, and answer any questions that participants might have. Lifetime risk and remaining risk estimates were provided to participants in oral, written, and visual formats; educational materials included risk curves showing participants' lifetime $\mathrm{AD}$ risk as compared to other first-degree relatives and the general population that were developed specifically for this study. ${ }^{23}$ Participants were also given a take-home letter summarizing their study results. Lifetime risk estimates provided to participants ranged from $13 \%$ to $57 \%$ and were based on data from a 17-center program of genetic epidemiology studies of $\mathrm{AD}$ based at Boston University.724-25 Participants were followed for one year after the Disclosure session (including 6-week, 6-month, and 1-year follow-up visits) for data collection and safety monitoring purposes.

\section{Measures}

Age, race, gender, years of education, annual household income, and number of affected relatives were assessed by selfreport. Worry about $\mathrm{AD}$ was assessed by asking participants to respond on a 5 -point Likert scale $(1=$ strongly agree to $5=$ strongly disagree) to the following item: "I am worried that I will develop Alzheimer's disease." The main outcome in data analyses was participation in the clinical trial.

\section{Data analyses}

Descriptive statistics were used to characterize the study sample and advancement through the study's prerandomization stages. Logistic regression analyses were used to assess demographic predictors of study participation among participants who completed the phone interview stage (earliest study stage at which data were collected). In these analyses, the following variables were included: age, gender, education, income, number of affected relatives, and worry about AD. Race was not included because of insufficient variability in the study sample. Cutpoints were chosen that approximated a median split in the data and/or represented a meaningful group dis- tinction. For example, we chose age 60 as a cutpoint because it represents the first decade of life when incidence of $\mathrm{AD}$ begins to increase significantly.

\section{RESULTS}

\section{Sample demographics}

Demographic characteristics of the systematically contacted and self-referred subsamples are presented in Table 1. Overall, both subsamples were predominantly White, highly educated, and of high socioeconomic status. Compared to the systematically contacted group, self-referred participants were slightly younger, of higher socioeconomic status, and more likely to be female. Self-referred participants were also slightly more likely to be unmarried and non-White.

\section{Study progression}

Data on participants' progression through each of the study's five stages are provided in Fig. 1. For this study, 196 participants were systematically contacted through their family's membership in AD research registries at the study sites. Of those, 86 declined to participate further in the study or to complete the brief demographic phone interview, leaving 110 participants (56\% of those contacted) who completed the phone interview. Of these participants, 60 went on to attend the education session to learn more about the study protocol. Of these, $48(80 \%)$ went on to attend the counseling/blood draw session. Then, 47 were ultimately randomized and received risk disclosure. Overall, 24\% (47/196) of all systematically contacted par-

Table 1

Demographic characteristics, by referral source

\begin{tabular}{|c|c|c|}
\hline Demographic characteristic & $\begin{array}{l}\text { Self-referred } \\
(n=179)\end{array}$ & $\begin{array}{l}\text { Systematically contacted } \\
\qquad(n=110)\end{array}$ \\
\hline $\begin{array}{l}\text { Mean Age }{ }^{a} \text {, Years (SD); } \\
\text { Range }\end{array}$ & $\begin{array}{l}52.5(9.0) \\
31-82\end{array}$ & $\begin{array}{l}57.9(12.4) \\
\quad 30-82\end{array}$ \\
\hline Gender $^{a}$, no. female (\%) & $141 \quad(78.8 \%)$ & $64 \quad(58.2 \%)$ \\
\hline Race/ethnicity ${ }^{b}$, no. White (\%) & $163(91.1 \%)$ & $107 \quad(97.3 \%)$ \\
\hline $\begin{array}{l}\text { Mean Years of education }{ }^{a} \\
\text { (SD); } \\
\text { Range }\end{array}$ & $16.7(2.3)$ & $15.4(2.5)$ \\
\hline $\begin{array}{l}\text { Marital status }{ }^{b}, \text { no. married } \\
\quad(\%)\end{array}$ & $110 \quad(61.4 \%)$ & $80 \quad(72.7 \%)$ \\
\hline \multicolumn{3}{|l|}{ No. of affected relatives (\%) } \\
\hline $\begin{array}{l}1 \\
2+\end{array}$ & $\begin{aligned} 77 & (43 \%) \\
102 & (57 \%)\end{aligned}$ & $\begin{array}{ll}49 & (44.5 \%) \\
61 & (55.5 \%)\end{array}$ \\
\hline \multicolumn{3}{|l|}{ Site $^{a}$, no. participants (\%) } \\
\hline $\begin{array}{l}\text { Boston } \\
\text { Cornell } \\
\text { Case Western }\end{array}$ & $\begin{array}{ll}64 & (35.7 \%) \\
88 & (49.2 \%) \\
27 & (15.1 \%)\end{array}$ & $\begin{aligned} 48 & (43.6 \%) \\
3 & (2.7 \%) \\
59 & (53.6 \%)\end{aligned}$ \\
\hline Median income bracket & $\$ 70 \mathrm{~K}-\$ 99,999$ & $\$ 50 \mathrm{~K}-\$ 69,999$ \\
\hline Worried about $\mathrm{AD}^{a}$ (\% agree) & $77 \%$ & $53 \%$ \\
\hline
\end{tabular}




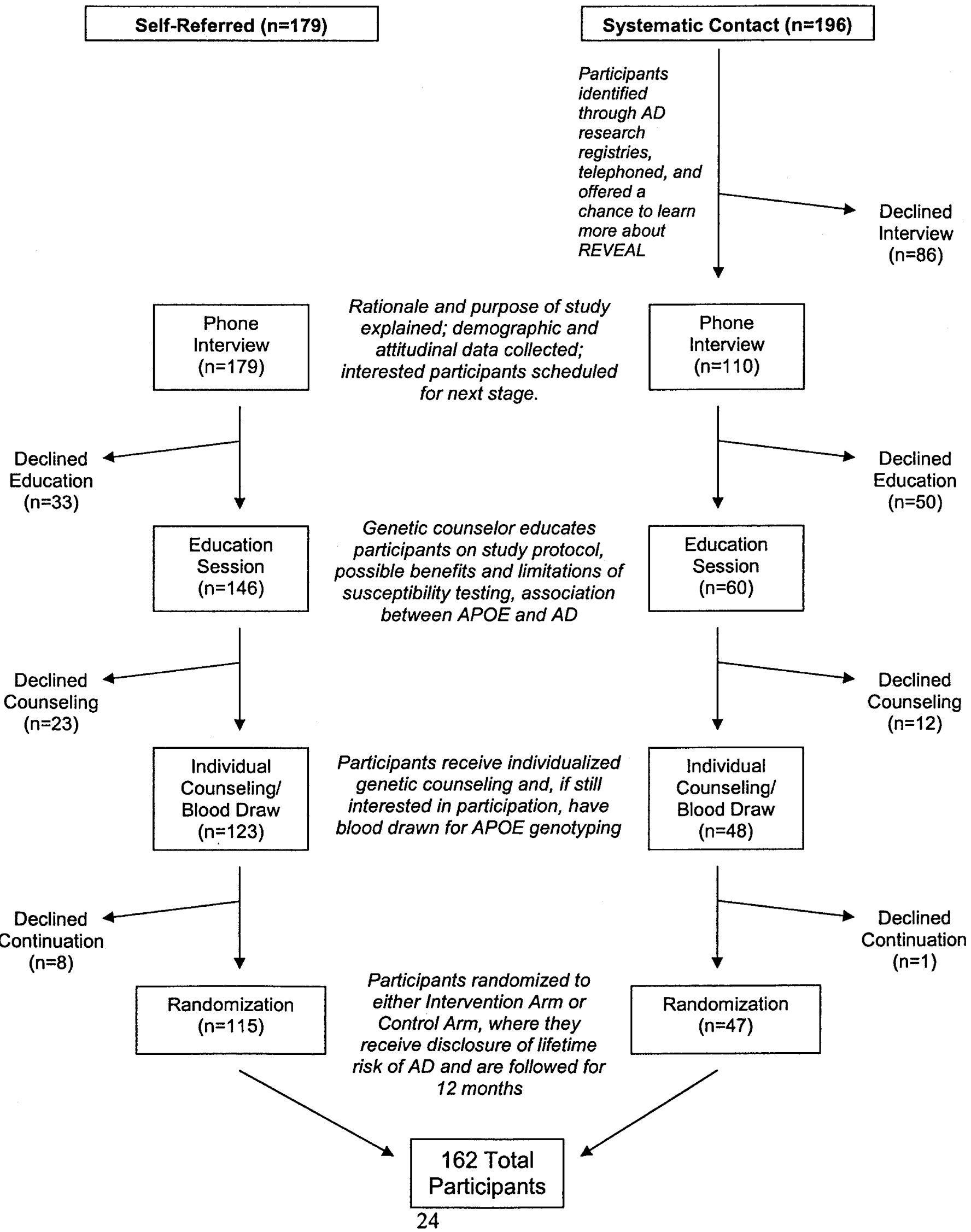

Fig. 1. Participants' progression through prerandomization phases. 
ticipants proceeded from initial contact all the way through to enrollment in the clinical trial, where they received disclosure of $\mathrm{AD}$ risk.

Of the 179 participants who were self-referred, 146 (82\%) attended the education session. Of these, $123(84 \%)$ went on to attend the counseling/blood draw session. Of these, 115 (93\%) were ultimately randomized into the clinical trial. Overall, $64 \%$ $(115 / 179)$ of self-referred participants advanced all the way from the initial phone interview to enrollment in the clinical trial. A review of genetic counselor notes from participant visits showed the following reasons for declining to continue in the study: concerns about emotional well-being; demands of study participation (e.g., too much time, travel); lack of prevention options for $\mathrm{AD}$; limitations of test information; lack of interest; personal or family health problems; and not wanting to be randomized to a control group. Certain concerns were more prominent before the Education Session phases of the study (e.g., lack of interest, personal or family health problems), whereas others were more prominent after the Education Session (e.g., limitations of test information, not wanting to be randomized to a control group). Although this study focuses on the study's prerandomization and enrollment phases, it should be noted that 160 of 162 RCT participants (99\%) completed at least one follow-up visit, and 147 of 162 (91\%) completed the year-long follow-up protocol.

\section{Predictors of test-seeking}

Results of the logistic regression analyses are presented in Table 2. Seven participants were excluded from these analyses due to missing data on at least one of their predictor variables. Significant predictors of participation in the clinical trial were age below 60 and college graduate education status $(P<0.01)$. Gender, income level, and number of affected relatives were not associated with participation in the clinical trial.

\section{DISCUSSION}

This is the first study to assess uptake of genetic testing for Alzheimer's disease. Nearly a quarter of all systematically contacted participants, and over $60 \%$ of self-referred participants, proceeded from initial contact to randomization into the study. Nearly $80 \%$ of participants in both groups who attended the study's initial education session went on to participate in the clinical trial. These groups maintained an interest in genetic susceptibility testing despite formal education about the absence of prevention options for $\mathrm{AD}$ and the relative uncertainty of test information. The one-quarter figure is at the high end of the range of reported test uptake rates in disorders without effective prevention or treatment options, but it is lower than reported uptake rates for disorders with more effective prevention or treatment options. For example, uptake rates for predictive testing for Huntington's disease have generally been estimated at below $15 \% .^{19,26} \mathrm{~A}$ study of cystic fibrosis screening within an HMO population found overall uptake rates of $4 \%$ to $23 \%$, depending on how testing was offered. ${ }^{27}$ Of eligible first-degree relatives, 30\% accepted susceptibility testing in a
Table 2

Predictors of participation in the clinical trial

\begin{tabular}{|c|c|c|}
\hline \multirow[b]{2}{*}{ Variable } & \multicolumn{2}{|c|}{$\begin{array}{l}\% \text { proceeding from initial contact to } \\
\text { clinical trial participation, by recruitment } \\
\text { method }\end{array}$} \\
\hline & Systematically contacted & Self-referred \\
\hline \multicolumn{3}{|l|}{ Age } \\
\hline Under age 60 & $51 \%$ & $64 \%$ \\
\hline Age 60 and above & $30 \%$ & $65 \%$ \\
\hline Adjusted odds ratio (95\% CI) & $3.80^{a}(1.37,10.53)$ & $1.02(0.37,2.81)$ \\
\hline \multicolumn{3}{|l|}{ Gender } \\
\hline Female & $45 \%$ & $62 \%$ \\
\hline Male & $39 \%$ & $71 \%$ \\
\hline Adjusted odds ratio (95\% CI) & $1.01(0.41,2.48)$ & $1.18(0.48,2.91)$ \\
\hline \multicolumn{3}{|l|}{ Education } \\
\hline College graduates & $52 \%$ & $67 \%$ \\
\hline Did not graduate college & $31 \%$ & $56 \%$ \\
\hline Adjusted odds ratio $(95 \% \mathrm{CI})$ & $3.47^{a}(1.37,8.78)$ & $1.23(0.55,2.77)$ \\
\hline \multicolumn{3}{|l|}{ Income } \\
\hline$\$ 70,000$ and above & $42 \%$ & $74 \%$ \\
\hline Below $\$ 70,000$ & $43 \%$ & $57 \%$ \\
\hline Adjusted odds ratio $(95 \% \mathrm{CI})$ & $0.47(0.18,1.25)$ & $2.20(0.95,5.06)$ \\
\hline \multicolumn{3}{|l|}{ Marital status } \\
\hline Married & $44 \%$ & $63 \%$ \\
\hline Not married & $43 \%$ & $57 \%$ \\
\hline Adjusted odds ratio (95\% CI) & $1.27(0.46,3.50)$ & $0.86(0.38,1.94)$ \\
\hline \multicolumn{3}{|l|}{ Number of affected relatives } \\
\hline$>1$ affected relative & $44 \%$ & $67 \%$ \\
\hline 1 affected relative & $41 \%$ & $61 \%$ \\
\hline Adjusted odds ratio $(95 \% \mathrm{CI})$ & $0.96(0.40,2.28)$ & $0.88(0.42,1.86)$ \\
\hline \multicolumn{3}{|l|}{ Worried about AD } \\
\hline Agree & $47 \%$ & $67 \%$ \\
\hline Other & $38 \%$ & $54 \%$ \\
\hline Adjusted odds ratio (95\% CI) & $1.11(0.44,2.81)$ & $2.27^{a}(1.03,5.00)$ \\
\hline
\end{tabular}

hereditary nonpolyposis colorectal cancer study. ${ }^{28}$ A $43 \%$ uptake rate was observed in a prospective study of BRCA1 testing among members of families affected by breast-ovarian cancer. ${ }^{29}$ Finally, uptake rates for predictive testing for treatable familial cancer syndromes (e.g., familial adenomatous polyposis and von Hippel-Lindau disease) have been estimated at $85 \% .{ }^{30}$ Most of these studies, like ours, relied on research registries for recruitment. Table 3 summarizes the current findings on test uptake in relation to testing for these other disorders.

The large difference in uptake rates between the systematically contacted and self-referred groups was not surprising, given the disparate ways in which these groups were accessed. The self-referred group approached our study with a priori interest in participation, whereas the systematically contacted group did not. Thus, the self-referred group was likely more intrinsically motivated to seek susceptibility testing than the systematically contacted group, perhaps propelled by the greater concern about developing $\mathrm{AD}$ that this group demon- 
Table 3

Summary of genetic test uptake rates, by disorder

\begin{tabular}{|c|c|c|c|c|}
\hline Disorder & Usual age of onset & Type of testing & $\begin{array}{l}\text { Effective prevention/ } \\
\text { treatment options? }\end{array}$ & $\begin{array}{l}\text { Estimated } \\
\text { uptake rate }\end{array}$ \\
\hline Familial adenomatous polyposis & Adulthood & Predictive & Yes & $85 \%{ }^{30}$ \\
\hline Breast-ovarian cancer & Adulthood & Susceptibility & Yes & $43 \%{ }^{29}$ \\
\hline Hereditary nonpolyposis colorectal cancer & Adulthood & Susceptibility & Yes & $30 \%{ }^{28}$ \\
\hline Alzheimer's disease & Late adulthood & Susceptibility & No & $24 \%$ \\
\hline Cystic fibrosis & Childhood & Carrier screening & No & $4 \%-23 \% 27$ \\
\hline Huntington's disease & Adulthood & Predictive & No & $10 \%^{31}$ \\
\hline
\end{tabular}

strated at baseline (worry about $\mathrm{AD}$ was a significant predictor of study participation among self-referred participants). The self-referred group may be the more appropriate of the two subsamples to consider when thinking about who might come forward for susceptibility testing in a future risk assessment context. However, the systematically contacted group may be more appropriate to consider when thinking about how many at-risk individuals might pursue risk assessment for $\mathrm{AD}$, given that we had a more meaningful denominator in this subsample from which to estimate participation rates.

The proportion of participants seeking testing in the present study was striking for two main reasons. First, susceptibility testing for $\mathrm{AD}$ could potentially be of interest to millions of people who have a parent affected by the disease. Indeed, $\mathrm{AD}$ is generally much more common than other disorders for which genetic testing takes place, with an incidence rate twice that of breast cancer, the next most common disease with genetic testing options..$^{15}$ Second, significant interest in testing occurred despite the fact that test information was relatively ambiguous and even though there are currently no treatments to prevent or cure $\mathrm{AD}$. Why would someone be interested in genetic testing if results do not impact medical care for the disease? A related study of ours suggested that participants endorsed numerous reasons for pursuing testing, including (1) to contribute to research ( $94 \%$ of participants endorsing), (2) to inform arrangements of personal affairs $(87 \%),(3)$ the hope that effective treatments will be developed (87\%), (4) to inform arrangements for long-term care (81\%), (5) to help prepare their family for their possible future illness (78\%), and (6) to do things sooner than they had otherwise planned (75\%). ${ }^{32}$ In addition, while participants' response to testing will be the subject of forthcoming articles, our preliminary results suggest that the vast majority of RCT participants did not experience adverse psychological effects and found the risk assessment experience worthwhile. ${ }^{33-34}$ Taken together, these findings suggest that a significant number of adult children of people with $\mathrm{AD}$ will be interested in pursuing genetic susceptibility testing and for many nonmedical reasons.

We also found that age below 60 and college education level predicted test seeking among systematically contacted participants. Furthermore, self-referred participants were also likely to be below age 60 and college educated. The age finding may reflect the fact that the planning issues cited by participants as important motivations to seek testing are most prominent in middle age. Cohort differences may also help explain this finding, as "baby boomers" tend to be more health informationseeking than their older counterparts. Age differences in test uptake have not generally been reported in other predictive genetic testing contexts, where average age of disease onset is much younger than in $\mathrm{AD}$ and thus age ranges of test candidates are much more restricted. Our finding that higher education level predicted test seeking is consistent with research on test uptake in HD. ${ }^{31}$ Although gender was not a predictor of advancement to randomization in this study, women represented the vast majority of self-referred participants. This finding is consistent with research in other disease contexts, where women have been more likely to pursue testing than men. ${ }^{35}$ As in testing for several other diseases, pretest worry was a significant predictor of test interest.

\section{Study limitations}

The study had several limitations that must be taken into account when interpreting results. First, the study population differed from the general population at-risk for $\mathrm{AD}$ as it was predominantly White, of high socioeconomic status, and had a particular interest in research. Although race and possession of health insurance have been suggested as demographic predictors of test interest in other studies, our sample lacked sufficient variability on these characteristics to conduct analyses. Another factor limiting generalizability was that our sample may have had different reasons for pursuing testing (perhaps most importantly, to contribute to research) than will test candidates in an actual clinical setting. The study obtained a NIH Certificate of Confidentiality protecting participants' test information; this safeguard may have encouraged study participation and would likely not be available in general clinical settings.

Previous research has found that rates of test uptake can vary considerably according to how the test is offered. ${ }^{27}$ Our results may have differed had we chosen either a less persuasive (mail only) or more persuasive (in-person interviews) strategy for initial contact. Another limitation of the study was that data on several predictors of test interest were not available at the initial stage of recruitment because they were obtained via a phone 
interview to which not all participants agreed. As such, predictors of interest were only valid from the phone interview stage forward.

\section{Conclusions}

Genetic susceptibility testing for common, complex diseases is likely to become an increasingly relevant risk assessment option. Research such as the REVEAL Study can help anticipate response to such testing in order to inform development of requisite education and counseling programs for at-risk participants. It remains to be seen how and when genetic risk assessment for Alzheimer's disease will ultimately be incorporated into clinical care, and for a variety of reasons one cannot assume that findings from this study will necessarily generalize to future scenarios. However, if interest in genetic susceptibility testing is even a fraction of what was expressed in this study, then demand for such services may strain available educational and counseling resources. Future research in this area is therefore clearly necessary, and it should look beyond demographic predictors to individual characteristics (e.g., illness perceptions, attitudes toward genetic testing, health psychological styles) that are likely to shape interest in and response to genetic susceptibility testing. ${ }^{15,35}$

\section{ACKNOWLEDGMENTS}

This study was supported by the following National Institutes of Health grants: National Human Genome Research Institute/National Institute on Aging (NIA) grant R01 HG/ AG02213 (The REVEAL Study), NIA grant 2 P30 AG13846 (Boston University Alzheimer's Disease Center), NIA grant R01 AG09029 (The MIRAGE Study), and National Center for Research Resources grant M01 RR00533 (Boston University Medical Campus General Clinical Research Center).

\section{References}

1. Caspi A, Sugden K, Moffitt TE et al. Influence of life stress on depression: Moderation by a polymorphism in the 5-HTT gene. Science 2003;301:386-389.

2. Gretarsdottir S, Thorleifsson G, Reynisdottir ST et al. The gene encoding phosphodiesterase 4D confers risk of ischemic stroke. Nat Genet 2003;35:131-138.

3. Masters CL, Beyreuther K. Science, medicine, and the future: Alzheimer's disease. BMJ 1998;316:446-448.

4. Roses AD. Genetic testing for Alzheimer disease: practical and ethical issues. Arch Neurol 1997;54:1226-1229.

5. Blacker D, Tanzi RE. The genetics of Alzheimer disease: current status and future prospects. Arch Neurol 1998;55:294-296.

6. St. George-Hyslop PH, Farrer LA, Goedert M. Alzheimer disease and the frontotemporal dementias: diseases with cerebral deposition of fibrillar proteins. In: Scriver C et al., eds. Molecular and metabolic basis of inherited disease, 8 th ed. Columbus, OH: McGraw-Hill, 2000:4:5785-5899.

7. Farrer LA, Cupples LA, Haines JL et al. Effects of age, sex and ethnicity on the association between apolipoprotein E genotype and Alzheimer's disease: A metaanalysis. JAMA 1997;278:1349-1356.

8. Karlinsky H, Sadovnick AD, Burgess MM, Lnglois S, Hayden MR, Berg JM. Issues in molecular genetic testing of individuals with suspected early-onset familial Alzheimer's disease. Alzheimer Dis Assoc Disord 1994;8:116-125.
9. Green RC. Genetic testing for Alzheimer's disease: has the moment arrived? Alz Care Quarterly 2002; 208-214.

10. Brodaty H, Conneally M, Gauthier S, Jennings C, Lennox A, Lovestone S. Consensus statement on predictive testing for Alzheimer disease. Alzheimer Dis Assoc Disord 1995;9:182-187.

11. Farrer LA, Brin MF, Elsas L et al. Statement on use of apolipoprotein E testing for Alzheimer disease. JAMA 1995;274:1627-1629.

12. Relkin NR, National Institute on Aging/Alzheimer's Association Working Group. Apolipoprotein E genotyping in Alzheimer's disease. Lancet 1996;347:1091-1095.

13. Post SG, Whitehouse PJ, Binstock RH et al. The clinical introduction of genetic testing for Alzheimer disease: an ethical perspective. JAMA 1997;277:832-836.

14. McConnell LM, Koenig BA, Greely HT, Raffin TA, and the Alzheimer Disease Working Group of the Stanford Program in Genomics, Ethics, \& Society. Genetic testing and Alzheimer disease: has the time come? Nat Med 1998:4:757-759.

15. Roberts JS. Anticipating response to predictive genetic testing for Alzheimer's disease: a survey of first-degree relatives. Gerontologist 2000;40:43-52.

16. Green RC, Clarke VC, Thompson NJ, Woodard JL, Letz R. Early detection of Alzheimer disease: methods, markers, and misgivings. Alzheimer Dis Assoc Disord 1997; 11(suppl 5):S1-S5.

17. Hipps YG, Roberts JS, Farrer LA, Green RC. Differences between African Americans and Whites in their attitudes toward genetic testing for Alzheimer's disease. Genet Test 2003;7:39-44.

18. Neumann PJ, Hammitt JK, Mueller C et al. Public attitudes about genetic testing for Alzheimer's disease. Health Aff 2001;20:252-264.

19. Quaid KA, Morris M. Reluctance to undergo predictive testing: the case of Huntington disease. Am J Med Genet 1993;45:41-45.

20. Randolph C. Repeatable Battery for the Assessment of Neuropsychological Status Standardization Edition Manual. San Antonio, TX: The Psychological Corporation, 1995.

21. Radloff LS. The CES-D Scale: A self report depression scale for research in the general population. Appl Psychol Measur 1977;1:385-401.

22. Beck AT, Steer RA. Beck Depression Inventory. New York: The Psychological Corporation, Harcourt Brace Javanovich, Inc.; 1987.

23. Cupples LA, Farrer LA, Sadovnick AD et al. Estimating risk curves for first-degree relatives of patients with Alzheimer's disease: The REVEAL Study. Genet Med 2004, 6:000-000.

24. Green RC, Cupples LA, Go R et al. Risk of dementia among white and African American relatives of Alzheimer's disease patients. JAMA 2002;287:329-336.

25. Lautenschlager NT, Cupples LA, Rao VS et al. Risk of dementia among relatives of Alzheimer's disease patients in the MIRAGE study: What is in store for the oldest old? Neurology 1996;46:641-650.

26. Craufurd D, Kerzin-Storrer L, Dodge A, Harris R. Uptake of presymptomatic predictive testing for Huntington's disease. Lancet 1989:603-605.

27. Tambor ES, Bernhardt BA, Chase GA et al. Offering cystic fibrosis carrier screening to an HMO population: Factors associated with utilization. Am J Hum Genet 1994; 55:626-637.

28. Codori AM, Petersen GM, Miglioretti DL et al. Attitudes toward colon cancer gene testing: factors predicting test uptake. Cancer Epidemiol Biomarkers Prev 1999;8: 345-351.

29. Lerman C, Narod S, Schulman K et al. BRCA1 testing in families with hereditary breast-ovarian cancer. JAMA 1996;275:1885-1892.

30. Evans D, Gareth R, Maher ER et al. Uptake of genetic testing for cancer predisposition. J Med Genet 1997;34:746-748.

31. Meiser B, Dunn S. Psychological impact of genetic testing for Huntington's disease: an update of the literature. J Neurol Neurosurg Psychiatry 2000;69:574-578.

32. Roberts JS, LaRusse SA, Katzen $\mathrm{H}$ et al. Reasons for seeking genetic susceptibility testing among first-degree relatives of people with Alzheimer disease. Alzheimer Dis Assoc Disord 2003;17:86-93.

33. Brown TC, Roberts JS, LaRusse SA et al. Impact of genetic risk assessment for Alzheimer disease. J Genet Counseling 2002;11:446-447.

34. Roberts JS, Green RC, Relkin NR et al. How do participants rate the impact of genetic susceptibility testing for Alzheimer's disease? Neurology 2003;60:A453.

35. Marteau TM, Croyle RT. The new genetics: Psychological responses to genetic testing. BMJ 1998;316:693-696. 